\title{
Revisting the Hodges-Lehmann estimator in a location mixture model: Is asymptotic normality good enough? (Supplementary material)
}

\author{
FADOUA BALABDAOUI
}

Seminar für Statistik, ETH Zürich, 8092, Zürich, Schweiz

E-mail: fadoua.balabdaoui@stat.math.ethz.ch

Proof of Theorem 2.6. In the sequel, we will write $F$ for $F^{0}$. In the sequel, we shall use the well-known fact that there exists a Brownian Bridge $\mathbb{U}$ defined on the same probability space as $\mathbb{F}_{n}$ such that for all $x \in \mathbb{R}$ we have that

$$
\mathbb{F}_{n}(x)=F(x)+\frac{\mathbb{U}_{n}(F(x))}{\sqrt{n}}
$$

and $\left\|\mathbb{U}_{n}-\mathbb{U}\right\|_{\infty} \rightarrow 0$ almost surely as $n \rightarrow \infty$. We will show a stronger version of convergence than the one presented in the theorem. More precisely, we will show that

$$
\sup _{h \in[-K, K]^{3}}\left|\mathbb{Q}_{n}(h)-\mathbb{Q}^{0}(h)\right|=0
$$

almost surely where $\mathbb{Q}^{0}$ is a function of the special Brownian Bridge $\mathbb{U}$ considered at the beginning of this proof. 
Uniform convergence of $T_{n, 3}(h)$ : We have that

$$
\begin{aligned}
& \sup _{h \in[-K, K]^{3}} \mid \int_{\mathbb{R}}\left(\mathbb{F}_{n}\left(\mu_{2, n, h_{2}}^{0}-t\right)+\mathbb{F}_{n}\left(\mu_{2, n, h_{2}}^{0}+t\right)-\mathbb{F}_{n}\left(\mu_{1, n, h_{1}}^{0}-t\right)-\mathbb{F}_{n}\left(\mu_{1, n, h_{1}}^{0}+t\right)\right)^{2} d t \\
& \quad-\int_{\mathbb{R}}\left(F\left(\mu_{2, n, h_{2}}^{0}-t\right)+F\left(\mu_{2, n, h_{2}}^{0}+t\right)-F\left(\mu_{1, n, h_{1}}^{0}-t\right)-F\left(\mu_{1, n, h_{1}}^{0}+t\right)\right)^{2} d t \mid \\
& \leq \sup _{h \in[-K, K]^{3}} 8\left(\int_{\mathbb{R}}\left|\mathbb{F}_{n}\left(\mu_{1, n, h_{1}}^{0}-t\right)-F\left(\mu_{1, n, h_{1}}^{0}-t\right)\right| d t\right. \\
& \quad+\int_{\mathbb{R}}\left|\mathbb{F}_{n}\left(\mu_{1, n, h_{1}}^{0}+t\right)-F\left(\mu_{1, n, h_{1}}^{0}+t\right)\right| d t \\
& \quad+\int_{\mathbb{R}}\left|\mathbb{F}_{n}\left(\mu_{2, n, h_{2}}^{0}-t\right)-F\left(\mu_{2, n, h_{2}}^{0}-t\right)\right| d t \\
& \left.\quad+\int_{\mathbb{R}}\left|\mathbb{F}_{n}\left(\mu_{2, n, h_{2}}^{0}+t\right)-F\left(\mu_{2, n, h_{2}}^{0}+t\right)\right| d t\right) \\
& \leq 32 \int_{\mathbb{R}}\left|\mathbb{F}_{n}(x)-F^{0}(x)\right| d x \rightarrow 0, \text { almost surely }
\end{aligned}
$$

as shown by Hunter et al. (2007); see the proof of their Lemma 1. Now,

$$
\begin{aligned}
& \sup _{h \in[-K, K]^{3}} \mid \int_{\mathbb{R}}\left(F\left(\mu_{2, n, h_{2}}^{0}-t\right)+F\left(\mu_{2, n, h_{2}}^{0}+t\right)-F\left(\mu_{1, n, h_{1}}^{0}-t\right)-F\left(\mu_{1, n, h_{1}}^{0}+t\right)\right)^{2} d t \\
& \quad-\int_{\mathbb{R}}\left(F\left(\mu_{2}^{0}-t\right)+F\left(\mu_{2}^{0}+t\right)-F\left(\mu_{1}^{0}-t\right)-F\left(\mu_{1}^{0}+t\right)\right)^{2} d t \mid \\
& \leq \frac{8}{\sqrt{n}} \sup _{h \in[-K, K]^{3}} \int_{\mathbb{R}}\left(h_{2}\left(f\left(\theta_{n, t, 3}\right)+f\left(\theta_{n, t, 4}\right)\right)-h_{1}\left(f\left(\theta_{n, t, 1}\right)+f\left(\theta_{n, t, 2}\right)\right)\right) d t \\
& \leq \frac{8 K}{\sqrt{n}} \sup _{h \in[-K, K]^{3}} \int_{\mathbb{R}}\left(f\left(\theta_{n, t, 1}\right)+f\left(\theta_{n, t, 2}+f\left(\theta_{n, t, 3}\right)+f\left(\theta_{n, t, 4}\right)\right) d t\right.
\end{aligned}
$$

where $\theta_{n, t, 1}$ and $\theta_{n, t, 2}$ are real numbers between $\mu_{1}^{0}-t$ and $\mu_{1}^{0}+h_{1} / \sqrt{n}-t$ and $\mu_{1}^{0}+t$ and $\mu_{1}^{0}+h_{1} / \sqrt{n}+t$ respectively. Similarly, $\theta_{n, t, 3}$ and $\theta_{n, t, 4}$ are real numbers between $\mu_{2}^{0}-t$ and $\mu_{2}^{0}+h_{2} / \sqrt{n}-t$ and $\mu_{2}^{0}+t$ and $\mu_{2}^{0}+h_{2} / \sqrt{n}+t$ respectively. Now note that $\left|\mu_{1}^{0}+h_{1} / \sqrt{n}-t-\left(\mu_{1}^{0}-t\right)\right| \leq K / \sqrt{n} \rightarrow 0$ so that $\theta_{n, t, 1} \in\left[\mu_{1}^{0}-t-\delta_{0}, \mu_{1}^{0}-t+\delta_{0}\right]$ for some $\delta_{0}>0$. This $\delta_{0}$ can be taken small enough so that $g$ is either increasing or decreasing on $\left[-t-\delta_{0},-t+\delta_{0}\right]$ and $\left[\mu_{1}^{0}-\mu_{2}^{0}-t-\delta_{0}, \mu_{1}^{0}-\mu_{2}^{0}-t+\delta_{0}\right]$. Hence, for $n$ large enough we have that

$$
f\left(\theta_{n, t, 1}\right) \leq g\left(-t-\delta_{0}\right)+g\left(-t+\delta_{0}\right)+g\left(\mu_{1}^{0}-\mu_{2}^{0}-t-\delta_{0}\right)+g\left(\mu_{1}^{0}-\mu_{2}^{0}-t+\delta_{0}\right)
$$

which is an integrable function. Using a similar reasoning, we conclude that the third term converges to 0 , and

$\sup _{h \in[-K, K]^{3}}\left|T_{n, 3}(h)-h_{3}^{2} \int_{\mathbb{R}}\left(F\left(\mu_{2}^{0}-t\right)+F\left(\mu_{2}^{0}+t\right)-F\left(\mu_{1}^{0}-t\right)-F\left(\mu_{1}^{0}+t\right)\right)^{2} d t\right| \rightarrow 0$ 
almost surely.

Uniform convergence of $T_{n, 2}(h)$. We can write

$$
\begin{aligned}
& \int_{\mathbb{R}} \sqrt{n}\{ \pi^{0}\left(1-\mathbb{F}_{n}\left(\mu_{1, n, h_{1}}^{0}-t\right)-\mathbb{F}_{n}\left(\mu_{1, n, h_{1}}^{0}+t\right)\right) \\
&+\left.\left(1-\pi^{0}\right)\left(1-\mathbb{F}_{n}\left(\mu_{2, n, h_{2}}^{0}-t\right)-\mathbb{F}_{n}\left(\mu_{2, n, h_{2}}^{0}+t\right)\right)\right\} \\
& \times\left(\mathbb{F}_{n}\left(\mu_{2, n, h_{2}}^{0}-t\right)+\mathbb{F}_{n}\left(\mu_{2, n, h_{2}}^{0}+t\right)-\mathbb{F}_{n}\left(\mu_{1, n, h_{1}}^{0}-t\right)-\mathbb{F}_{n}\left(\mu_{1, n, h_{1}}^{0}+t\right)\right) d t \\
&=-\int_{\mathbb{R}}\left\{\pi^{0}\left(\mathbb{U}_{n}\left(F\left(\mu_{1, n, h_{1}}^{0}-t\right)\right)+\mathbb{U}_{n}\left(F\left(\mu_{1, n, h_{1}}^{0}+t\right)\right)\right)\right. \\
&+\left.\left(1-\pi^{0}\right)\left(\mathbb{U}_{n}\left(F\left(\mu_{2, n, h_{2}}^{0}-t\right)\right)+\mathbb{U}_{n}\left(F\left(\mu_{2, n, h_{2}}^{0}+t\right)\right)\right)\right\} \\
& \times\left(\mathbb{F}_{n}\left(\mu_{2, n, h_{2}}^{0}-t\right)+\mathbb{F}_{n}\left(\mu_{2, n, h_{2}}^{0}+t\right)-\mathbb{F}_{n}\left(\mu_{1, n, h_{1}}^{0}-t\right)-\mathbb{F}_{n}\left(\mu_{1, n, h_{1}}^{0}+t\right)\right) d t \\
&+\int_{\mathbb{R}} \sqrt{n}\left\{\pi^{0}\left(1-F\left(\mu_{1, n, h_{1}}^{0}-t\right)-F\left(\mu_{1, n, h_{1}}^{0}+t\right)\right)\right. \\
&\left.\quad+\left(1-\pi^{0}\right)\left(1-F\left(\mu_{2, n, h_{2}}^{0}-t\right)-F\left(\mu_{2, n, h_{2}}^{0}+t\right)\right)\right\} \\
& \quad \times\left(\mathbb{F}_{n}\left(\mu_{2, n, h_{2}}^{0}-t\right)+\mathbb{F}_{n}\left(\mu_{2, n, h_{2}}^{0}+t\right)-\mathbb{F}_{n}\left(\mu_{1, n, h_{1}}^{0}-t\right)-\mathbb{F}_{n}\left(\mu_{1, n, h_{1}}^{0}+t\right)\right) d t \\
&=-A_{1}\left(h_{1}, h_{2}\right)+A_{2}\left(h_{1}, h_{2}\right) .
\end{aligned}
$$

Note that we have dropped the subscript $n$ to have a less cumbersome notation. Let us denote $\Delta_{n}(x)=\mathbb{U}_{n}(x)-\mathbb{U}(x)$ for $x \in[0,1]$. Then, we have

$$
A_{1}\left(h_{1}, h_{2}\right)=A_{1,1}\left(h_{1}, h_{2}\right)+A_{1,2}\left(h_{1}, h_{2}\right)+A_{1,3}\left(h_{1}, h_{2}\right)+A_{1,4}\left(h_{1}, h_{2}\right)
$$

where

$$
\begin{aligned}
& A_{1,1}\left(h_{1}, h_{2}\right) \\
& =\int_{\mathbb{R}}\left\{\pi^{0}\left(\mathbb{U}\left(F\left(\mu_{1, n, h_{1}}^{0}-t\right)\right)+\mathbb{U}\left(F\left(\mu_{1, n, h_{1}}^{0}+t\right)\right)\right)\right. \\
& \left.\quad+\left(1-\pi^{0}\right)\left(\mathbb{U}\left(F\left(\mu_{2, n, h_{2}}^{0}-t\right)\right)+\mathbb{U}\left(F\left(\mu_{2, n, h_{2}}^{0}+t\right)\right)\right)\right\} \\
& \quad \times\left(F\left(\mu_{2, n, h_{2}}^{0}-t\right)+F\left(\mu_{2, n, h_{2}}^{0}+t\right)-F\left(\mu_{1, n, h_{1}}^{0}-t\right)-F\left(\mu_{1, n, h_{1}}^{0}+t\right)\right) d t,
\end{aligned}
$$

$$
\begin{aligned}
& A_{1,2}\left(h_{1}, h_{2}\right) \\
& =\int_{\mathbb{R}}\left\{\pi^{0}\left(\mathbb{U}\left(F\left(\mu_{1, n, h_{1}}^{0}-t\right)\right)+\mathbb{U}\left(F\left(\mu_{1, n, h_{1}}^{0}+t\right)\right)\right)\right. \\
& \left.\quad+\left(1-\pi^{0}\right)\left(\mathbb{U}\left(F\left(\mu_{2, n, h_{2}}^{0}-t\right)\right)+\mathbb{U}\left(F\left(\mu_{2, n, h_{2}}^{0}+t\right)\right)\right)\right\} \\
& \quad \times\left(\left(\mathbb{F}_{n}\left(\mu_{2, n, h_{2}}^{0}-t\right)-F\left(\mu_{2, n, h_{2}}^{0}-t\right)\right)+\left(\mathbb{F}_{n}\left(\mu_{2, n, h_{2}}^{0}+t\right)-F\left(\mu_{2, n, h_{2}}^{0}+t\right)\right)\right. \\
& \left.\quad+\left(\mathbb{F}_{n}\left(\mu_{1, n, h_{1}}^{0}-t\right)-F\left(\mu_{1, n, h_{1}}^{0}-t\right)\right)+\left(\mathbb{F}_{n}\left(\mu_{1, n, h_{1}}^{0}+t\right)-F\left(\mu_{1, n, h_{1}}^{0}+t\right)\right)\right) d t,
\end{aligned}
$$




$$
\begin{aligned}
& A_{1,3}\left(h_{1}, h_{2}\right) \\
& =\int_{\mathbb{R}}\left\{\pi^{0}\left(\Delta\left(F\left(\mu_{1, n, h_{1}}^{0}-t\right)\right)+\Delta\left(F\left(\mu_{1, n, h_{1}}^{0}+t\right)\right)\right)\right. \\
& \quad+\left(1-\pi^{0}\right)\left(\Delta\left(F\left(\mu_{2, n, h_{2}}^{0}-t\right)\right)+\Delta\left(F\left(\mu_{2, n, h_{2}}^{0}+t\right)\right)\right) \\
& \quad \times\left(\left(\mathbb{F}_{n}\left(\mu_{2, n, h_{2}}^{0}-t\right)-F\left(\mu_{2, n, h_{2}}^{0}-t\right)\right)+\left(\mathbb{F}_{n}\left(\mu_{2, n, h_{2}}^{0}+t\right)-F\left(\mu_{2, n, h_{2}}^{0}+t\right)\right)\right. \\
& \left.\quad+\left(\mathbb{F}_{n}\left(\mu_{1, n, h_{1}}^{0}-t\right)-F\left(\mu_{1, n, h_{1}}^{0}-t\right)\right)+\left(\mathbb{F}_{n}\left(\mu_{1, n, h_{1}}^{0}+t\right)-F\left(\mu_{1, n, h_{1}}^{0}+t\right)\right)\right) d t,
\end{aligned}
$$

and

$$
\begin{aligned}
& A_{1,4}\left(h_{1}, h_{2}\right) \\
& =\int_{\mathbb{R}}\left\{\pi^{0}\left(\Delta\left(F\left(\mu_{1, n, h_{1}}^{0}-t\right)\right)+\Delta\left(F\left(\mu_{1, n, h_{1}}^{0}+t\right)\right)\right)\right. \\
& \quad+\left(1-\pi^{0}\right)\left(\Delta\left(F\left(\mu_{2, n, h_{2}}^{0}-t\right)\right)+\Delta\left(F\left(\mu_{2, n, h_{2}}^{0}+t\right)\right)\right) \\
& \quad \times\left(F\left(\mu_{2, n, h_{2}}^{0}-t\right)+F\left(\mu_{2, n, h_{2}}^{0}+t\right)-F\left(\mu_{1, n, h_{1}}^{0}-t\right)-F\left(\mu_{1, n, h_{1}}^{0}+t\right)\right) d t .
\end{aligned}
$$

Now we have that

$$
\sup _{\left(h_{1}, h_{2}\right) \in[-K, K]^{2}}\left|A_{1,2}\left(h_{1}, h_{2}\right)\right| \leq 8\|U\|_{\infty} \int_{\mathbb{R}}\left|\mathbb{F}_{n}(x)-F(x)\right| d x \rightarrow 0
$$

and

$$
\sup _{\left(h_{1}, h_{2}\right) \in[-K, K]^{2}}\left|A_{1,3}\left(h_{1}, h_{2}\right)\right| \leq 8\left\|\mathbb{U}_{n}-\mathbb{U}\right\|_{\infty} \int_{\mathbb{R}}\left|\mathbb{F}_{n}(x)-F(x)\right| d x \rightarrow 0
$$

almost surely. Also,

$$
\begin{aligned}
& \sup _{\left(h_{1}, h_{2}\right) \in[-K, K]^{2}}\left|A_{1,4}\left(h_{1}, h_{2}\right)\right| \\
& \leq 2\left\|\mathbb{U}_{n}-\mathbb{U}\right\|_{\infty}\left(\int_{\mathbb{R}}\left|F\left(\mu_{2, n, h_{2}}^{0}-t\right)-F\left(\mu_{1, n, h_{1}}^{0}-t\right)\right| d t\right. \\
& \left.\quad+\int_{\mathbb{R}}\left|F\left(\mu_{2, n, h_{2}}^{0}+t\right)-F\left(\mu_{1, n, h_{1}}^{0}+t\right)\right| d t\right) \\
& \leq 2\left\|\mathbb{U}_{n}-\mathbb{U}\right\|_{\infty}\left(\int_{\mathbb{R}}\left(F\left(\mu_{2}^{0}+1 / 2-t\right)-F\left(\mu_{1}^{0}-1 / 2-t\right)\right) d t\right. \\
& \left.\quad+\int_{\mathbb{R}}\left(F\left(\mu_{2}^{0}+1 / 2+t\right)-F\left(\mu_{1}^{0}-1 / 2+t\right)\right) d t\right),
\end{aligned}
$$

for $n$ large enough

$$
\rightarrow 0
$$


almost surely. Let

$$
\begin{aligned}
L=\int_{\mathbb{R}} & \left\{\pi^{0}\left(\mathbb{U}\left(F\left(\mu_{1}^{0}-t\right)\right)+\mathbb{U}\left(F\left(\mu_{1}^{0}+t\right)\right)\right)+\left(1-\pi^{0}\right)\left(\mathbb{U}\left(F\left(\mu_{2}^{0}-t\right)\right)+\mathbb{U}\left(F\left(\mu_{2}^{0}+t\right)\right)\right)\right\} \\
\times & \left(F\left(\mu_{2}^{0}-t\right)+F\left(\mu_{2}^{0}+t\right)-F\left(\mu_{1}^{0}-t\right)-F\left(\mu_{1}^{0}+t\right)\right) d t .
\end{aligned}
$$

Now, we will show that

$$
\sup _{\left(h_{1}, h_{2}\right) \in[-K, K]^{2}}\left|A_{1,1}\left(h_{1}, h_{2}\right)-L\right| \rightarrow 0
$$

almost surely. Let us write $\mathbb{U}(a, b)=\mathbb{U}(b)-\mathbb{U}(a)$, and $F(a, b)=F(b)-F(a)$. Then, we can write

$$
\begin{aligned}
A_{1,1}\left(h_{1}, h_{2}\right)-L \\
=\int_{\mathbb{R}}\left\{\pi^{0}\left(\mathbb{U}\left(F\left(\mu_{1}^{0}-t\right), F\left(\mu_{1, n, h_{1}}^{0}-t\right)\right)+\mathbb{U}\left(F\left(\mu_{1}^{0}+t\right), F\left(\mu_{1, n, h_{1}}^{0}+t\right)\right)\right)\right. \\
\left.+\quad\left(1-\pi^{0}\right)\left(\mathbb{U}\left(F\left(\mu_{2}^{0}-t\right), F\left(\mu_{2, n, h_{2}}^{0}-t\right)\right)+\mathbb{U}\left(F\left(\mu_{2}^{0}+t\right), F\left(\mu_{2, n, h_{2}}^{0}+t\right)\right)\right)\right\} \\
\quad \times\left(F\left(\mu_{2, n, h_{2}}^{0}-t\right)+F\left(\mu_{2, n, h_{2}}^{0}+t\right)-F\left(\mu_{1, n, h_{1}}^{0}-t\right)-F\left(\mu_{1, n, h_{1}}^{0}+t\right)\right) d t \\
+\int_{\mathbb{R}}\left\{\pi^{0}\left(\mathbb{U}\left(F\left(\mu_{1}^{0}-t\right)\right)+\mathbb{U}\left(F\left(\mu_{1}^{0}+t\right)\right)\right)+\left(1-\pi^{0}\right)\left(\mathbb{U}\left(F\left(\mu_{2}^{0}-t\right)\right)+\mathbb{U}\left(F\left(\mu_{2}^{0}+t\right)\right)\right)\right\} \\
\quad \times\left(F\left(\mu_{2}^{0}-t, \mu_{2, n, h_{2}}^{0}-t\right)+F\left(\mu_{2}^{0}+t, \mu_{2, n, h_{2}}^{0}+t\right)\right. \\
\left.\quad \quad-F\left(\mu_{1}^{0}-t, \mu_{1, n, h_{1}}^{0}-t\right)-F\left(\mu_{1}^{0}+t, \mu_{1, n, h_{1}}^{0}+t\right)\right) d t .
\end{aligned}
$$

Now, note that Taylor expansion of order 1 of $F$ and the fact that $\|f\|_{\infty} \leq g(0)$ imply that

$$
\left|F\left(\mu_{j}^{0} \pm t, \mu_{j, n, h_{j}}^{0} \pm t\right)\right| \leq \frac{g(0)\left|h_{j}\right|}{\sqrt{n}} \leq \frac{g(0) K}{\sqrt{n}}:=a_{n}
$$

for $j=1,2$. Now, it follows from Lévy's theorem (see e.g. see Shorack and Wellner (1986)) that

$$
\begin{aligned}
\sup _{|t-s| \leq a_{n}}|\mathbb{U}(s, t)| & \leq \frac{3}{2} \sqrt{a_{n} \log \left(1 / a_{n}\right)} \\
& \leq \frac{3 D^{1 / 2}}{2 \sqrt{2}} \frac{(\log n)^{1 / 2}}{n^{1 / 4}}, \text { for } n>D^{2}
\end{aligned}
$$


with $D=\max (g(0) K, 1)$. This implies that we have almost surely

$$
\begin{aligned}
& \sup _{\left(h_{1}, h_{2}\right) \in[-K, K]^{2}} \mid \int_{\mathbb{R}}\left\{\pi ^ { 0 } \left(\mathbb { U } \left(F\left(\mu_{1}^{0}-t\right), F\left(\mu_{1, n, h_{1}}^{0}-t\right)+\mathbb{U}\left(F\left(\mu_{1}^{0}+t\right), F\left(\mu_{1, n, h_{1}}^{0}+t\right)\right)\right.\right.\right. \\
&+\left(1-\pi^{0}\right)\left(\mathbb{U}\left(F\left(\mu_{2}^{0}-t\right), F\left(\mu_{2, n, h_{2}}^{0}-t\right)+\mathbb{U}\left(F\left(\mu_{2}^{0}+t\right), F\left(\mu_{2, n, h_{2}}^{0}+t\right)\right)\right\}\right. \\
& \times\left(F\left(\mu_{2, n, h_{2}}^{0}-t\right)+F\left(\mu_{2, n, h_{2}}^{0}+t\right)-F\left(\mu_{1, n, h_{1}}^{0}-t\right)-F\left(\mu_{1, n, h_{1}}^{0}+t\right)\right) d t \mid \\
& \leq \frac{3 D(\log n)^{1 / 2}}{2 \sqrt{2} n^{1 / 4}}\left\{\int_{\mathbb{R}}\left(F\left(\mu_{2}^{0}+1 / 2-t\right)-F\left(\mu_{1}^{0}-1 / 2-t\right)\right)\right. \\
&\left.+\left(F\left(\mu_{2}^{0}+1 / 2+t\right)-F\left(\mu_{1}^{0}-1 / 2+t\right)\right) d t\right\}, \text { for } n \text { large enough. }
\end{aligned}
$$

Using again Taylor expansion of $F$ as done in (0.1), we have that

$$
\begin{aligned}
& \mid \int_{\mathbb{R}}\left\{\pi^{0}\left(\mathbb{U}\left(F\left(\mu_{1}^{0}-t\right)\right)+\mathbb{U}\left(F\left(\mu_{1}^{0}+t\right)\right)\right)+\left(1-\pi^{0}\right)\left(\mathbb{U}\left(F\left(\mu_{2}^{0}-t\right)\right)+\mathbb{U}\left(F\left(\mu_{2}^{0}+t\right)\right)\right)\right\} \\
& \times\left(F\left(\mu_{2}^{0}-t, \mu_{2, n, h_{2}}^{0}-t\right)+F\left(\mu_{2}^{0}+t, \mu_{2, n, h_{2}}^{0}+t\right)\right. \\
& \left.-F\left(\mu_{1}^{0}-t, \mu_{1, n, h_{1}}^{0}-t\right)-F\left(\mu_{1}^{0}+t, \mu_{1, n, h_{1}}^{0}+t\right)\right) d t \mid \\
& \leq \frac{2\|U\|_{\infty}}{\sqrt{n}} \int_{\mathbb{R}}\left\{\left|h_{2}\right|\left(f\left(\theta_{n, t, 3}\right)+f\left(\theta_{n, t, 4}\right)\right)+\left|h_{1}\right|\left(f\left(\theta_{n, t, 1}\right)+f\left(\theta_{n, t, 2}\right)\right)\right\} d t \\
& \leq \frac{2 K\|U\|_{\infty}}{\sqrt{n}} \int_{\mathbb{R}}\left\{f\left(\theta_{n, t, 1}\right)+f\left(\theta_{n, t, 2}\right)+f\left(\theta_{n, t, 3}\right)+f\left(\theta_{n, t, 4}\right)\right\} d t
\end{aligned}
$$

where the integrand can be bounded by an integrable function independent of $h_{1}, h_{2}$ as argued above. It follows that both terms in the upper bound of $A_{1,1}\left(h_{1}, h_{2}\right)-L$ converge to 0 uniformly almost surely, and hence $\sup _{\left(h_{1}, h_{2}\right) \in[-K, K]^{2}}\left|A_{1,1}\left(h_{1}, h_{2}\right)-L\right|=0$ almost surely.

Now, we tackle $A_{2}$. We recall that for all $t \in \mathbb{R}$

$$
\pi^{0}\left(1-F\left(\mu_{1}^{0}-t\right)-F\left(\mu_{1}^{0}+t\right)\right)+\left(1-\pi^{0}\right)\left(1-F\left(\mu_{2}^{0}-t\right)-F\left(\mu_{2}^{0}+t\right)\right)=0 .
$$

Hence, using Taylor expansion up to the second order, we can write

$$
\begin{aligned}
& \sqrt{n}\left\{\pi^{0}\left(1-F\left(\mu_{1, n, h_{1}}^{0}-t\right)-F\left(\mu_{1, n, h_{1}}^{0}+t\right)\right)+\left(1-\pi^{0}\right)\left(1-F\left(\mu_{2, n, h_{2}}^{0}-t\right)\right.\right. \\
& \left.\left.\quad-F\left(\mu_{2, n, h_{2}}^{0}+t\right)\right)\right\} \\
& =-h_{1} \pi^{0}\left(f\left(\mu_{1}^{0}-t\right)+f\left(\mu_{1}^{0}+t\right)\right)-h_{2}\left(1-\pi^{0}\right)\left(f\left(\mu_{2}^{0}-t\right)+f\left(\mu_{2}^{0}+t\right)\right) \\
& \quad-\frac{h_{1}^{2} \pi^{0}}{2 \sqrt{n}}\left(f^{\prime}\left(\tilde{\theta}_{n, t, 1}\right)+f^{\prime}\left(\tilde{\theta}_{n, t, 2}\right)\right)-\frac{h_{2}^{2}\left(1-\pi^{0}\right)}{2 \sqrt{n}}\left(f^{\prime}\left(\tilde{\theta}_{n, t, 3}\right)+f^{\prime}\left(\tilde{\theta}_{n, t, 4}\right)\right)
\end{aligned}
$$


where $\tilde{\theta}_{n, t, j}$ is between $\mu_{1}^{0} \pm t$ and $\mu_{1}^{0}+h_{1} / \sqrt{n} \pm t$ for $j=1,2$ and $\tilde{\theta}_{n, t, j}$ is between $\mu_{2}^{0} \pm t$ and $\mu_{2}^{0}+h_{2} / \sqrt{n} \pm t$ for $j=3$, 4 . Let

$$
\begin{aligned}
L^{\prime}\left(h_{1}, h_{2}\right)=- & \int_{\mathbb{R}}\left(h_{1} \pi^{0}\left(f\left(\mu_{1}^{0}-t\right)+f\left(\mu_{1}^{0}+t\right)\right)+h_{2}\left(1-\pi^{0}\right)\left(f\left(\mu_{2}^{0}-t\right)+f\left(\mu_{2}^{0}+t\right)\right)\right. \\
& \times\left(F\left(\mu_{2}^{0}-t\right)+F\left(\mu_{2}^{0}+t\right)-F\left(\mu_{1}^{0}-t\right)-F\left(\mu_{1}^{0}+t\right)\right) d t .
\end{aligned}
$$

Then, using the fact that $f$ and $f^{\prime}$ are bounded on $\mathbb{R}$ by some $M>0$ we have for $n$ large enough that

$$
\begin{aligned}
& \sup _{\left(h_{1}, h_{2}\right) \in[-K, K]^{2}}\left|A_{2}\left(h_{1}, h_{2}\right)-L^{\prime}\left(h_{1}, h_{2}\right)\right| \\
& \leq 16 M K(1+K) \int_{\mathbb{R}}\left|\mathbb{F}_{n}(x)-F(x)\right| d x+\frac{16 M K^{2}}{\sqrt{n}} \\
& +\frac{2 K^{2} M}{\sqrt{n}}\left\{\int_{\mathbb{R}}\left(F\left(\mu_{2}^{0}+1 / 2-t\right)-F\left(\mu_{1}^{0}-1 / 2-t\right)\right) d t\right. \\
& \left.+\int_{\mathbb{R}}\left(F\left(\mu_{2}^{0}+1 / 2+t\right)-F\left(\mu_{1}^{0}-1 / 2+t\right)\right) d t\right\} \\
& \rightarrow 0
\end{aligned}
$$

almost surely. Since $h_{3} \in[-K, K]$, we conclude from the calculations above that $T_{n, 2}(h)$ converges uniformly to

$$
\begin{aligned}
-2 h_{3}\left\{\int_{\mathbb{R}}\right. & \left\{\pi ^ { 0 } \left(\mathbb{U}\left(F\left(\mu_{1}^{0}-t\right)\right)+\mathbb{U}\left(F\left(\mu_{1}^{0}+t\right)\right)\right.\right. \\
& \left.+\left(1-\pi^{0}\right)\left(\mathbb{U}\left(F\left(\mu_{2}^{0}-t\right)\right)+\mathbb{U}\left(F\left(\mu_{2}^{0}+t\right)\right)\right)\right\} \\
& \times\left(F\left(\mu_{2}^{0}-t\right)+F\left(\mu_{2}^{0}+t\right)-F\left(\mu_{1}^{0}-t\right)-F\left(\mu_{1}^{0}+t\right)\right) d t \\
+ & \int_{\mathbb{R}}\left(h_{1} \pi^{0}\left(f\left(\mu_{1}^{0}-t\right)+f\left(\mu_{1}^{0}+t\right)\right)+h_{2}\left(1-\pi^{0}\right)\left(f\left(\mu_{2}^{0}-t\right)+f\left(\mu_{2}^{0}+t\right)\right)\right. \\
& \left.\times\left(F\left(\mu_{2}^{0}-t\right)+F\left(\mu_{2}^{0}+t\right)-F\left(\mu_{1}^{0}-t\right)-F\left(\mu_{1}^{0}+t\right)\right) d t\right\}
\end{aligned}
$$

almost surely on $[-K, K]^{3}$.

Uniform convergence of $T_{n, 1}\left(h_{1}, h_{2}\right)$. Using the Brownian bridge approximation, we can write

$$
T_{n, 1}\left(h_{1}, h_{2}\right)=B_{1}\left(h_{1}, h_{2}\right)+B_{2}\left(h_{1}, h_{2}\right)+B_{3}\left(h_{1}, h_{2}\right)
$$


where

$$
\begin{aligned}
& B_{1}\left(h_{1}, h_{2}\right)=n \int_{\mathbb{R}}\{ \pi^{0}\left(1-F\left(\mu_{1, n, h_{1}}^{0}-t\right)-F\left(\mu_{1, n, h_{1}}^{0}+t\right)\right) \\
&+\left.\left(1-\pi^{0}\right)\left(1-F\left(\mu_{2, n, h_{2}}^{0}-t\right)-F\left(\mu_{2, n, h_{2}}^{0}+t\right)\right)\right\}^{2} d t \\
& B_{2}\left(h_{1}, h_{2}\right)=-2 \sqrt{n} \int_{\mathbb{R}}\left\{\pi^{0}\left(1-F\left(\mu_{1, n, h_{1}}^{0}-t\right)-F\left(\mu_{1, n, h_{1}}^{0}+t\right)\right)\right. \\
&\left.+\left(1-\pi^{0}\right)\left(1-F\left(\mu_{2, n, h_{2}}^{0}-t\right)-F\left(\mu_{2, n, h_{2}}^{0}+t\right)\right)\right\} \\
& \times\left\{\pi ^ { 0 } \left(\mathbb { U } _ { n } \left(F\left(\mu_{1, n, h_{1}}^{0}-t\right)+\mathbb{U}_{n}\left(F\left(\mu_{1, n, h_{1}}^{0}+t\right)\right)\right.\right.\right. \\
&+\left(1-\pi^{0}\right)\left(\mathbb{U}_{n}\left(F\left(\mu_{2, n, h_{2}}^{0}-t\right)+\mathbb{U}_{n}\left(F\left(\mu_{1, n, h_{2}}^{0}+t\right)\right)\right\} d t\right.
\end{aligned}
$$

and

$$
\begin{aligned}
& B_{3}\left(h_{1}, h_{2}\right)=\int_{\mathbb{R}}\left\{\pi ^ { 0 } \left(\mathbb { U } _ { n } \left(F\left(\mu_{1, n, h_{1}}^{0}-t\right)+\mathbb{U}_{n}\left(F\left(\mu_{1, n, h_{1}}^{0}+t\right)\right)\right.\right.\right. \\
&+\left(1-\pi^{0}\right)\left(\mathbb{U}_{n}\left(F\left(\mu_{2, n, h_{2}}^{0}-t\right)+\mathbb{U}_{n}\left(F\left(\mu_{1, n, h_{2}}^{0}+t\right)\right)\right\}^{2} d t .\right.
\end{aligned}
$$

Taylor expansion up to the second order yields

$$
\begin{aligned}
& B_{1}\left(h_{1}, h_{2}\right) \\
& =n \int_{\mathbb{R}}\left\{-\frac{h_{1}}{\sqrt{n}} \pi^{0}\left(f\left(\mu_{1}^{0}-t\right)+f\left(\mu_{1}^{0}+t\right)\right)-\frac{h_{2}}{\sqrt{n}}\left(1-\pi^{0}\right)\left(f\left(\mu_{2}^{0}-t\right)+f\left(\mu_{2}^{0}+t\right)\right)\right. \\
& \left.-\frac{h_{1}^{2}}{2 n} \pi^{0}\left(f^{\prime}\left(\theta_{n, t, 1}\right)+f^{\prime}\left(\theta_{n, t, 2}\right)\right)-\frac{h_{2}^{2}}{2 n}\left(1-\pi^{0}\right)\left(f^{\prime}\left(\theta_{n, t, 3}\right)+f^{\prime}\left(\theta_{n, t, 4}\right)\right)\right\}^{2} \\
& =\int_{\mathbb{R}}\left(h_{1} \pi^{0}\left(f\left(\mu_{1}^{0}-t\right)+f\left(\mu_{1}^{0}+t\right)\right)+h_{2}\left(1-\pi^{0}\right)\left(f\left(\mu_{2}^{0}-t\right)+f\left(\mu_{2}^{0}+t\right)\right)\right)^{2} d t \\
& +\frac{1}{\sqrt{n}} \int_{\mathbb{R}}\left(h_{1} \pi^{0}\left(f\left(\mu_{1}^{0}-t\right)+f\left(\mu_{1}^{0}+t\right)\right)+h_{2}\left(1-\pi^{0}\right)\left(f\left(\mu_{2}^{0}-t\right)+f\left(\mu_{2}^{0}+t\right)\right)\right) \\
& \times\left(h_{1}^{2} \pi^{0}\left(f^{\prime}\left(\theta_{n, t, 1}\right)+f^{\prime}\left(\theta_{n, t, 2}\right)\right)+h_{2}^{2}\left(1-\pi^{0}\right)\left(f^{\prime}\left(\theta_{n, t, 3}\right)+f^{\prime}\left(\theta_{n, t, 4}\right)\right)\right) d t \\
& +\frac{1}{4 n} \int_{\mathbb{R}}\left(h_{1}^{2} \pi^{0}\left(f^{\prime}\left(\tilde{\theta}_{n, t, 1}\right)+f^{\prime}\left(\tilde{\theta}_{n, t, 2}\right)\right)+h_{2}^{2}\left(1-\pi^{0}\right)\left(f^{\prime}\left(\tilde{\theta}_{n, t, 3}\right)+f^{\prime}\left(\tilde{\theta}_{n, t, 4}\right)\right)\right)^{2} d t \\
& =\tilde{L}\left(h_{1}, h_{2}\right)+B_{1,1}\left(h_{1}, h_{2}\right)+B_{1,2}\left(h_{1}, h_{2}\right) / 4
\end{aligned}
$$


where $\tilde{\theta}_{n, t, j}$ for $j=1,2,3,4$ are defined above in (0.5). The goal now is to show that

$$
\sup _{\left(h_{1}, h_{2}\right) \in[-K, K]^{2}}\left|B_{1}\left(h_{1}, h_{2}\right)-\tilde{L}\left(h_{1}, h_{2}\right)\right| \rightarrow 0
$$

almost surely. Using the assumption that $\left|g^{\prime}\right|$ changes the direction of monotonicity finitely many times almost everywhere as this is true for $g$ and $g^{\prime}$, we can see easily that the same holds for $\left|f^{\prime}\right|$, and we have for $n$ large enough (independently of $h_{1}$ and $\left.h_{2}\right)$

$$
\begin{aligned}
& h_{1}^{2} \pi^{0}\left(\left|f^{\prime}\left(\theta_{n, t, 1}\right)\right|+\left|f^{\prime}\left(\theta_{n, t, 2}\right)\right|\right)+h_{2}^{2}\left(1-\pi^{0}\right)\left(\left|f^{\prime}\left(\theta_{n, t, 3}\right)\right|+\left|f^{\prime}\left(\theta_{n, t, 4}\right)\right|\right) \\
& \leq K^{2}\left(\left|f^{\prime}\left(\mu_{1}^{0}-t\right)\right|+\left|f^{\prime}\left(\mu_{1, n, h_{1}}^{0}-t\right)\right|+\left|f^{\prime}\left(\mu_{1}^{0}+t\right)\right|+\left|f\left(\mu_{1, n, h_{1}}^{0}+t\right)\right|\right. \\
& \left.\quad+\left|f^{\prime}\left(\mu_{2}^{0}-t\right)\right|+\left|f\left(\mu_{2, n, h_{2}}^{0}-t\right)\right|+\left|f^{\prime}\left(\mu_{2}^{0}+t\right)\right|+\left|f^{\prime}\left(\mu_{2, n, h_{2}}^{0}+t\right)\right|\right) .
\end{aligned}
$$

Using the fact that $\left|f^{\prime}\right|$ is bounded almost everywhere by some constant $M>0$, this implies that

$$
\begin{aligned}
& \sup _{\left(h_{1}, h_{2}\right) \in[-K, K]^{2}} \int_{\mathbb{R}}\left\{h_{1}^{2} \pi^{0}\left(\left|f^{\prime}\left(\theta_{n, t, 1}\right)\right|+\left|f^{\prime}\left(\theta_{n, t, 2}\right)\right|\right)\right. \\
& \left.+h_{2}^{2}\left(1-\pi^{0}\right)\left(\left|f^{\prime}\left(\theta_{n, t, 3}\right)\right|+\left|f^{\prime}\left(\theta_{n, t, 4}\right)\right|\right)\right\}^{2} d t \\
& \leq 16 K^{2} M \int_{\mathbb{R}}\left|f^{\prime}(t)\right| d t<\infty .
\end{aligned}
$$

That $\int_{\mathbb{R}}\left|f^{\prime}(t)\right| d t<\infty$ follows again from the fact that $f$ is bounded and that it changes direction of monotonicity only a finite number of times. This implies that $\left|f^{\prime}(t)\right|$ is either $f^{\prime}(t)$ or $-f^{\prime}(t)$ on a finite number of intervals. Hence, $\sup _{\left(h_{1}, h_{2}\right) \in[-K, K]^{2}}\left|B_{1,2}\left(h_{1}, h_{2}\right)\right| \rightarrow 0$ almost surely. Also, the function in the right-hand side in $B_{1,1}\left(h_{1}, h_{2}\right)$ is clearly integrable which implies $\sup _{\left(h_{1}, h_{2}\right) \in[-K, K]^{2}}\left|B_{1,1}\left(h_{1}, h_{2}\right)\right| \rightarrow 0$ almost surely using boundedness of $f^{\prime}$ as a consequence of boundedness of $g^{\prime}$. We conclude that the claimed uniform convergence 
in (0.6) is true. To tackle $B_{2}\left(h_{1}, h_{2}\right)$, we use again Taylor expansion of $F$ as done above:

$$
\begin{aligned}
& B_{2}\left(h_{1}, h_{2}\right) \\
& =2 \int_{\mathbb{R}}\left(h_{1} \pi^{0}\left(f\left(\mu_{1}^{0}-t\right)+f\left(\mu_{1}^{0}+t\right)\right)+h_{2}\left(1-\pi^{0}\right)\left(f\left(\mu_{2}^{0}-t\right)+f\left(\mu_{2}^{0}+t\right)\right)\right) \\
& \times\left\{\pi ^ { 0 } \left(\mathbb { U } _ { n } \left(F\left(\mu_{1, n, h_{1}}^{0}-t\right)+\mathbb{U}_{n}\left(F\left(\mu_{1, n, h_{1}}^{0}+t\right)\right)\right.\right.\right. \\
& \quad+\left(1-\pi^{0}\right)\left(\mathbb{U}_{n}\left(F\left(\mu_{2, n, h_{2}}^{0}-t\right)+\mathbb{U}_{n}\left(F\left(\mu_{1, n, h_{2}}^{0}+t\right)\right)\right\} d t\right. \\
& \left.+\frac{1}{\sqrt{n}} \int_{\mathbb{R}}\left(h_{1}^{2} \pi^{0}\left(f^{\prime}\left(\tilde{\theta}_{n, t, 1}\right)+f^{\prime}\left(\tilde{\theta}_{n, t, 2}\right)\right)+h_{2}^{2}\left(1-\pi^{0}\right)\left(f^{\prime}\left(\tilde{\theta}_{n, t, 3}\right)\right)+f^{\prime}\left(\tilde{\theta}_{n, t, 4}\right)\right)\right) \\
& \quad \times\left\{\pi ^ { 0 } \left(\mathbb { U } _ { n } \left(F\left(\mu_{1, n, h_{1}}^{0}-t\right)+\mathbb{U}_{n}\left(F\left(\mu_{1, n, h_{1}}^{0}+t\right)\right)\right.\right.\right. \\
& \quad+\left(1-\pi^{0}\right)\left(\mathbb{U}_{n}\left(F\left(\mu_{2, n, h_{2}}^{0}-t\right)+\mathbb{U}_{n}\left(F\left(\mu_{1, n, h_{2}}^{0}+t\right)\right)\right\} d t\right. \\
& =B_{2,1}\left(h_{1}, h_{2}\right)+B_{2,2}\left(h_{1}, h_{2}\right) .
\end{aligned}
$$

Note that

$$
\sup _{\left(h_{1}, h_{2}\right) \in[-K, K]^{2}}\left|B_{2,2}\left(h_{1}, h_{2}\right)\right| \leq \frac{8 K^{2}\|U\|_{\infty}}{\sqrt{n}} \int_{\mathbb{R}}\left|f^{\prime}\right| d t
$$

almost surely, where the argument goes along the lines of the one used above for $B_{1}$, and hence $\sup _{\left(h_{1}, h_{2}\right) \in[-K, K]^{2}}\left|B_{2,2}\left(h_{1}, h_{2}\right)\right| \rightarrow 0$ almost surely. Now let

$$
\begin{aligned}
& \tilde{L}^{\prime} \\
& =2 \int_{\mathbb{R}}\left(h_{1} \pi^{0}\left(f\left(\mu_{1}^{0}-t\right)+f\left(\mu_{1}^{0}+t\right)\right)+h_{2}\left(1-\pi^{0}\right)\left(f\left(\mu_{2}^{0}-t\right)+f\left(\mu_{2}^{0}+t\right)\right)\right) \\
& \quad \times\left\{\pi^{0}\left(\mathbb{U}\left(F\left(\mu_{1}^{0}-t\right)\right)+\mathbb{U}\left(F\left(\mu_{1}^{0}+t\right)\right)\right)+\left(1-\pi^{0}\right)\left(\mathbb{U}\left(F\left(\mu_{2}^{0}-t\right)\right)+\mathbb{U}\left(F\left(\mu_{2}^{0}+t\right)\right)\right)\right\} d t .
\end{aligned}
$$

We will show that

$$
\sup _{\left(h_{1}, h_{2}\right) \in[-K, K]^{2}}\left|B_{2,1}\left(h_{1}, h_{2}\right)-\tilde{L}^{\prime}\right| \rightarrow 0
$$

almost surely. But this goes along the proof of showing that $\sup _{\left(h_{1}, h_{2}\right) \in[-K, K]^{2}} \mid A_{1,1}\left(h_{1}, h_{2}\right)-$ $L \mid \rightarrow$ in (0.2). Finally, we will show that under the assumptions of the theorem we have that

$$
\sup _{\left(h_{1}, h_{2}\right) \in[-K, K]^{2}}\left|B_{3}\left(h_{1}, h_{2}\right)-\mathbb{B}\left(h_{1}, h_{2}\right)\right| \rightarrow 0
$$


almost surely where

$$
\begin{aligned}
\mathbb{B}\left(h_{1}, h_{2}\right)=\int_{\mathbb{R}}\{ & \pi^{0}\left(\mathbb{U}\left(F\left(\mu_{1}^{0}-t\right)\right)+\mathbb{U}\left(F\left(\mu_{1}^{0}+t\right)\right)\right) \\
& \left.+\left(1-\pi^{0}\right)\left(\mathbb{U}\left(F\left(\mu_{2}^{0}-t\right)\right)+\mathbb{U}\left(F\left(\mu_{2}^{0}-t\right)\right)\right)\right\}^{2} d t .
\end{aligned}
$$

With the special construction which allows us to use $\left\|\mathbb{U}_{n}-\mathbb{U}\right\|_{\infty} \rightarrow 0$ almost surely, we can write

$$
\begin{aligned}
& \left|B_{3}\left(h_{1}, h_{2}\right)-\mathbb{B}\left(h_{1}, h_{2}\right)\right| \\
& \leq\left(2\left\|\mathbb{U}_{n}-\mathbb{U}\right\|_{\infty}+\max _{j \in\{1,2\}} \sup _{t \in \mathbb{R}}\left|\mathbb{U}\left(F\left(\mu_{j, n, h_{j}}^{0} \pm t\right)\right)-\mathbb{U}\left(F\left(\mu_{j}^{0} \pm t\right)\right)\right|\right) \\
& \times\left\{\int_{\mathbb{R}}\left|\mathbb{U}_{n}\left(F\left(\mu_{1, n, h_{1}}^{0}-t\right)\right)+\mathbb{U}\left(F\left(\mu_{1}^{0}-t\right)\right)\right| d t+\int_{\mathbb{R}}\left|\mathbb{U}_{n}\left(F\left(\mu_{1, n, h_{1}}^{0}+t\right)\right)+\mathbb{U}\left(F\left(\mu_{1}^{0}+t\right)\right)\right| d t\right. \\
& \left.+\int_{\mathbb{R}}\left|\mathbb{U}_{n}\left(F\left(\mu_{1, n, h_{2}}^{0}-t\right)\right)+\mathbb{U}\left(F\left(\mu_{2}^{0}-t\right)\right)\right| d t+\int_{\mathbb{R}}\left|\mathbb{U}_{n}\left(F\left(\mu_{1, n, h_{2}}^{0}+t\right)\right)+\mathbb{U}\left(F\left(\mu_{2}^{0}+t\right)\right)\right| d t\right\} .
\end{aligned}
$$

Now note that (0.4) above allows to claim that

$$
\max _{j \in\{1,2\}} \sup _{t \in \mathbb{R}}\left|\mathbb{U}\left(F\left(\mu_{j, n, h_{j}}^{0} \pm t\right)\right)-\mathbb{U}\left(F\left(\mu_{j}^{0} \pm t\right)\right)\right| \leq \frac{3 D^{1 / 2}}{2 \sqrt{2}} \frac{(\log n)^{1 / 2}}{n^{1 / 4}}
$$

almost surely for all $n>D^{2}$ with $D=\max (g(0) K, 1)$. Also, note that

$$
\begin{aligned}
& \int_{\mathbb{R}}\left|\mathbb{U}\left(F\left(\mu_{1}^{0}-t\right)\right)\right| d t \\
& =\int_{\mathbb{R}} \frac{\left|\mathbb{U}\left(F\left(\mu_{1}^{0}-t\right)\right)\right|}{F\left(\mu_{1}^{0}-t\right)^{1 / 2-\alpha}\left(1-F\left(\mu_{1}^{0}-t\right)\right)^{1 / 2-\alpha}} F\left(\mu_{1}^{0}-t\right)^{1 / 2-\alpha}\left(1-F\left(\mu_{1}^{0}-t\right)\right)^{1 / 2-\alpha} d t
\end{aligned}
$$

where

$$
\begin{aligned}
& F\left(\mu_{1}^{0}-t\right)\left(1-F\left(\mu_{1}^{0}-t\right)\right) \\
& =\left(\pi^{0}\right)^{2} G\left(t-\mu_{1}^{0}\right)\left(1-G\left(t-\mu_{1}^{0}\right)\right) \\
& +\pi^{0}\left(1-\pi^{0}\right)\left[G\left(t-\mu_{1}^{0}\right)\left(1-G\left(t-\mu_{2}^{0}\right)\right)+G\left(t-\mu_{2}^{0}\right)\left(1-G\left(t-\mu_{1}^{0}\right)\right)\right] \\
& +\left(1-\pi^{0}\right)^{2} G\left(t-\mu_{2}^{0}\right)\left(1-G\left(t-\mu_{2}^{0}\right)\right) \\
& \leq\left[\left(\pi^{0}\right)^{2}+\pi^{0}\left(1-\pi^{0}\right)\right] G\left(t-\mu_{1}^{0}\right)\left(1-G\left(t-\mu_{1}^{0}\right)\right) \\
& +\left[\left(1-\pi^{0}\right)^{2}+\pi^{0}\left(1-\pi^{0}\right)\right] G\left(t-\mu_{2}^{0}\right)\left(1-G\left(t-\mu_{2}^{0}\right)\right), \\
& \quad \text { using } \mu_{1}^{0}<\mu_{2}^{0} \text { and monotonicity of } G
\end{aligned}
$$$$
\leq G\left(t-\mu_{1}^{0}\right)\left(1-G\left(t-\mu_{1}^{0}\right)\right)+G\left(t-\mu_{2}^{0}\right)\left(1-G\left(t-\mu_{2}^{0}\right)\right) \text {. }
$$ 
Now since $1 / 2-\alpha \in(0,1 / 2)$ the $c_{r}$ inequality for $r \in(0,1]$ yields

$$
\begin{aligned}
F\left(\mu_{1}^{0}-t\right)^{1 / 2-\alpha}\left(1-F\left(\mu_{1}^{0}-t\right)\right)^{1 / 2-\alpha} \leq & G\left(t-\mu_{1}^{0}\right)^{1 / 2-\alpha}\left(1-G\left(t-\mu_{1}^{0}\right)\right)^{1 / 2-\alpha} \\
& +G\left(t-\mu_{2}^{0}\right)^{1 / 2-\alpha}\left(1-G\left(t-\mu_{2}^{0}\right)\right)^{1 / 2-\alpha}
\end{aligned}
$$

which implies by assumption of the theorem that

$$
\int_{\mathbb{R}} F\left(\mu_{1}^{0}-t\right)^{1 / 2-\alpha}\left(1-F\left(\mu_{1}^{0}-t\right)\right)^{1 / 2-\alpha} d t<\infty
$$

Furthermore, the function $q(x)=[x(1-x)]^{1 / 2-\alpha}$ is symmetric around $1 / 2$, increasing on $\left[0,1 / 2\right.$ ), and $\int_{0}^{1} q^{-2}(x) d x<\infty$. It follows from Sen's inequality (see e.g. Shorack and Wellner (1986), page 139) that

$$
\begin{aligned}
& \int_{\mathbb{R}}\left|\mathbb{U}\left(F\left(\mu_{1}^{0}-t\right)\right)\right| d t \\
& \leq \sup _{t \in[0,1]} \frac{\left|\mathbb{U}\left(F\left(\mu_{1}^{0}-t\right)\right)\right|}{\left.F\left(\mu_{1}^{0}-t\right)\right)^{1 / 2-\alpha}\left(1-F\left(\mu_{1}^{0}-t\right)\right)^{1 / 2-\alpha}} \int_{\mathbb{R}} F\left(\mu_{1}^{0}-t\right)^{1 / 2-\alpha}\left(1-F\left(\mu_{1}^{0}-t\right)\right)^{1 / 2-\alpha} d t \\
& \leq \sup _{t \in[0,1]} \frac{|\mathbb{U}(x)|}{x^{1 / 2-\alpha}(1-x)^{1 / 2-\alpha}} \int_{\mathbb{R}} F\left(\mu_{1}^{0}-t\right)^{1 / 2-\alpha}\left(1-F\left(\mu_{1}^{0}-t\right)\right)^{1 / 2-\alpha} d t \\
& =\sup _{t \in[0,1]} \frac{|\mathbb{U}(x)|}{x^{1 / 2-\alpha}(1-x)^{1 / 2-\alpha}} \int_{\mathbb{R}} F(t)^{1 / 2-\alpha}(1-F(t))^{1 / 2-\alpha} d t
\end{aligned}
$$

almost surely. The same argument can be used for $\mathbb{U}\left(F\left(\mu_{1}^{0}+t\right)\right), \mathbb{U}\left(F\left(\mu_{2}^{0} \pm t\right)\right)$ and $\mathbb{U}_{n}\left(F\left(\mu_{j, n, h_{j}}^{0} \pm t\right)\right)-\mathbb{U}\left(F\left(\mu_{j}^{0} \pm t\right)\right)$ to conclude that

$$
\begin{aligned}
& \sup _{\left(h_{1}, h_{2}\right) \in[-K, K]^{2}}\left|B_{3}\left(h_{1}, h_{2}\right)-\mathbb{B}\left(h_{1}, h_{2}\right)\right| \\
& \leq 4\left(2\left\|\mathbb{U}_{n}-\mathbb{U}\right\|_{\infty}+\frac{9 D^{1 / 2}}{4} \frac{\log n}{n^{1 / 4}}\right) \\
& \quad \times\left(\sup _{t \in[0,1]} \frac{|\mathbb{U}(x)|}{x^{1 / 2-\alpha}(1-x)^{1 / 2-\alpha}}+\sup _{t \in[0,1]} \frac{\left|\mathbb{U}_{n}(x)-\mathbb{U}(x)\right|}{x^{1 / 2-\alpha}(1-x)^{1 / 2-\alpha}}\right) \\
& \quad \times \int_{\mathbb{R}} F(t)^{1 / 2-\alpha}(1-F(t))^{1 / 2-\alpha} d t .
\end{aligned}
$$


We conclude that $T_{n, 1}\left(h_{1}, h_{2}\right)$ converges almost surely to

$$
\begin{aligned}
\rightarrow & \int_{\mathbb{R}}\left(h_{1} \pi^{0}\left(f\left(\mu_{1}^{0}-t\right)+f\left(\mu_{1}^{0}+t\right)\right)+h_{2}\left(1-\pi^{0}\right)\left(f\left(\mu_{2}^{0}-t\right)+f\left(\mu_{2}^{0}+t\right)\right)\right)^{2} d t \\
+ & 2 \int_{\mathbb{R}}\left(h_{1} \pi^{0}\left(f\left(\mu_{1}^{0}-t\right)+f\left(\mu_{1}^{0}+t\right)\right)+h_{2}\left(1-\pi^{0}\right)\left(f\left(\mu_{2}^{0}-t\right)+f\left(\mu_{2}^{0}+t\right)\right)\right) \\
& \times\left\{\pi^{0}\left(\mathbb{U}\left(F\left(\mu_{1}^{0}-t\right)\right)+\mathbb{U}\left(F\left(\mu_{1}^{0}+t\right)\right)\right)+\left(1-\pi^{0}\right)\left(\mathbb{U}\left(F\left(\mu_{2}^{0}-t\right)\right)+\mathbb{U}\left(F\left(\mu_{2}^{0}+t\right)\right)\right)\right\} d t \\
+ & \int_{\mathbb{R}}\left\{\pi^{0}\left(\mathbb{U}\left(F\left(\mu_{1}^{0}-t\right)\right)+\mathbb{U}\left(F\left(\mu_{1}^{0}+t\right)\right)\right)+\left(1-\pi^{0}\right)\left(\mathbb{U}\left(F\left(\mu_{2}^{0}-t\right)\right)+\mathbb{U}\left(F\left(\mu_{2}^{0}-t\right)\right)\right)\right\}^{2} d t .
\end{aligned}
$$

uniformly on $[-K, K]^{2}$.

Let $\mathbb{Q}^{0}$ denote the limiting process of $\mathbb{Q}_{n}$. Using the formulae given below, it is easy to see that $\mathbb{Q}^{0}$ is given by the form of the statement of the theorem. Now, using the same special construction under which we have the almost sure convergence of the process $\mathbb{Q}_{n}(h)$ to $\mathbb{Q}^{0}(h)$, we can easily see that we have joint almost sure convergence

$$
\left.\left(\mathbb{Q}_{n}\left(h^{(1)}\right), \ldots, \mathbb{Q}_{n}\left(h^{(m)}\right)\right)\right) \rightarrow\left(\mathbb{Q}^{0}\left(h^{(1)}\right), \ldots, \mathbb{Q}^{0}\left(h^{(m)}\right)\right)
$$

for any integer $m \geq 2$, where $h^{(j)}$ are any vectors in $[-K, K]^{3}$.

It remains to show that $\mathbb{Q}_{n}$ is asymptotically tight. By Theorem 1.5.7 of van der Vaart and Wellner (1996), it is is enough to show that this process is asymptotically uniformly $\rho$-equicontinuous in probability, where we can take $\rho\left(h, h^{\prime}\right)=\left\|h^{\prime}-h\right\|_{\infty}$ (note that $\left([-K, K,]^{3}, \rho\right)$ is totally bounded). Thus, we need to show that for every $\epsilon, \eta>0$ there exists $\delta>0$ such that

$$
\limsup _{n \rightarrow \infty} P\left(\sup _{\left\|h^{\prime}-h\right\|_{\infty}<\delta}\left|\mathbb{Q}_{n}\left(h^{\prime}\right)-\mathbb{Q}_{n}(h)\right|>\epsilon\right)<\eta .
$$

Now, we have that

$$
\begin{aligned}
\left|\mathbb{Q}_{n}\left(h^{\prime}\right)-\mathbb{Q}_{n}(h)\right| & \leq\left|\mathbb{Q}_{n}\left(h^{\prime}\right)-\mathbb{Q}^{0}\left(h^{\prime}\right)\right|+\left|\mathbb{Q}^{0}\left(h^{\prime}\right)-\mathbb{Q}^{0}(h)\right|+\left|\mathbb{Q}(h)-\mathbb{Q}_{n}(h)\right| \\
& \leq 2 \sup _{h \in[-K, K]^{3}}\left|\mathbb{Q}_{n}(h)-\mathbb{Q}^{0}(h)\right|+\left|\mathbb{Q}^{0}\left(h^{\prime}\right)-\mathbb{Q}^{0}(h)\right| .
\end{aligned}
$$

Fix $\epsilon, \eta>0$. It follows from the calculations above that there exists an integer $n_{0}>0$ depending on $\epsilon$ and $\eta$ such that for $n \geq n_{0}$

$$
P\left(\sup _{h \in[-K, K]^{3}}\left|\mathbb{Q}_{n}(h)-\mathbb{Q}^{0}(h)\right|>\epsilon / 4\right)<\eta / 2 .
$$

Moreover, the function $h \mapsto h \mathbb{A} h^{T}=\psi(h)$ is clearly continuous in $h$ and hence uniformly continuous on the compact $[-K, K]^{3}$. Hence, there exists $\delta_{1}>0$ such that 
$\sup _{\left\|h^{\prime}-h\right\|_{\infty}<\delta_{1}}\left|\psi\left(h^{\prime}\right)-\psi(h)\right|<\epsilon / 4$. Also, if $S=\mathbb{V}_{1}+\mathbb{V}_{2}+\mathbb{V}_{3}$, then there exists $M>0$ depending on $\eta$ such that $P(|S|>M)<\eta / 4$. Hence,

$$
\begin{aligned}
P\left(\sup _{\left\|h^{\prime}-h\right\|_{\infty}<\delta_{2}}\left|\left(h^{\prime}-h\right) \mathbb{V}\right|>\epsilon / 4\right) & \leq P\left(\delta_{2}|S|>\epsilon / 4\right) \\
& <\eta / 2
\end{aligned}
$$

if we choose $\delta_{2}=\epsilon /(4 M)$. Now, take $\delta=\min \left(\delta_{1}, \delta_{2}\right)$ and $n>n_{0}$. Then, we have that

$$
\begin{aligned}
& P\left(\sup _{\left\|h^{\prime}-h\right\|_{\infty}<\delta}\left|\mathbb{Q}_{n}\left(h^{\prime}\right)-\mathbb{Q}_{n}(h)\right|>\epsilon\right) \\
& \leq P\left(2\left\|\mathbb{Q}_{n}-\mathbb{Q}^{0}\right\|_{[-K, K]^{3}}>\epsilon / 2\right) \\
& +P\left(\sup _{\left\|h^{\prime}-h\right\|_{\infty}<\delta}\left|\psi\left(h^{\prime}\right)-\psi(h)+\left(h^{\prime}-h\right) \mathbb{V}\right|>\epsilon / 2\right) \\
& <\eta / 2+P\left(\sup _{\left\|h^{\prime}-h\right\|_{\infty}<\delta}\left|\left(h^{\prime}-h\right) \mathbb{V}\right|>\epsilon / 4\right), \\
& <\eta / 2+P(|S| \geq M)<\eta .
\end{aligned}
$$

This completes the proof of the theorem.

Proof of Theorem 2.7. We first note that with the definition of the matrix $\mathbb{A}$ we have that

$$
\begin{array}{r}
h \mathbb{A} h^{T}=\int_{\mathbb{R}}\left\{\pi^{0}\left(f\left(\mu_{1}^{0}+t\right)+f\left(\mu_{1}^{0}-t\right)\right) h_{1}+\left(1-\pi^{0}\right)\left(f\left(\mu_{2}^{0}+t\right)+f\left(\mu_{2}^{0}-t\right)\right) h_{2}\right. \\
\left.-\left(F\left(\mu_{2}^{0}+t\right)-F\left(\mu_{1}^{0}+t\right)+F\left(\mu_{2}^{0}-t\right)-F\left(\mu_{1}^{0}-t\right)\right) h_{3}\right\}^{2}
\end{array}
$$

for any $h \in \mathbb{R}^{3}$ and hence $\mathbb{A}$ is semi-positive definite. To show that it is positive definite, we will show that under the assumptions of the theorem that $h \mathbb{A} h^{T}=0$ admits the unique solution $h=\underline{0}$. Now, the former equation is equivalent to having

$$
\begin{aligned}
& \pi^{0}\left(f\left(\mu_{1}^{0}+t\right)+f\left(\mu_{1}^{0}-t\right)\right) h_{1}+\left(1-\pi^{0}\right)\left(f\left(\mu_{2}^{0}+t\right)+f\left(\mu_{2}^{0}-t\right)\right) h_{2} \\
& =\left(F\left(\mu_{2}^{0}+t\right)-F\left(\mu_{1}^{0}+t\right)+F\left(\mu_{2}^{0}-t\right)-F\left(\mu_{1}^{0}-t\right)\right) h_{3}
\end{aligned}
$$

almost everywhere $t \in \mathbb{R}$. Using the notation of Appendix B, the identity in (0.7) can be rewritten as

$$
a g(t)+b \bar{g}_{s}(t)=c(G(t+s)-G(t-s))
$$

almost everywhere, where

$$
a=2 \pi^{0}\left(\pi^{0} h_{1}+\left(1-\pi^{0}\right) h_{2}\right), \quad b=2\left(1-\pi^{0}\right)\left(\left(1-\pi^{0}\right) h_{1}+\pi^{0} h_{2}\right), \quad c=h_{3} .
$$


Now, computing the moments of order 0,2 and 4 of both sides of (0.8) and using the formulae computed below yield

$$
\begin{aligned}
a+b & =2 s c \\
a \sigma^{2}+b\left(\sigma^{2}+s^{2}\right) & =c\left(2 \sigma^{2} s+\frac{2}{3} s^{3}\right), \\
a m_{4}+b\left(m_{4} s+6 \sigma^{2} s^{2}+s^{4}\right) & =2 c\left(m_{4} s+2 \sigma^{2} s^{3}+\frac{s^{5}}{5}\right)
\end{aligned}
$$

or equivalently

$$
\begin{aligned}
a+b & =2 s c \\
b & =\frac{2}{3} \delta s \\
b & =\frac{2\left(2 \sigma^{2}+s^{2} / 5\right)}{6 \sigma^{2}+s^{2}} \delta c .
\end{aligned}
$$

If $c \neq 0$, the second and third equations imply that $3 / 5 s^{2}=s^{2}$ which is impossible. We conclude that $a=b=c=0$ is the unique solution of the above linear system. The fact that $\pi^{0} \neq\{0,1 / 2,1\}$ implies in turn that $h_{1}=h_{2}=0$ so that we conclude that $h_{1}=h_{2}=h_{3}=0$. This completes the proof that $\mathbb{A}$ is positive definite. Now Proposition 3.5 and Theorem 3.6 allow us to apply the argmax continuous mapping theorem, e.g. Theorem 3.2.2 of van der Vaart and Wellner (1996) to conclude the result.

Some useful formulae. We start with some useful formulae. In the following, we write $g$ for $g^{0}, f$ for $f^{0}$ and $G$ for $G^{0}$ the CDF associated with $g$. Also, let

$$
s=u_{2}^{0}-u_{1}^{0}, \quad \text { and } \quad \bar{g}_{s}(x)=\frac{g(x-s)+g(x+s)}{2} .
$$

By symmetry of $g$ we have $g(-t)=g(t)$ and $G(-t)=1-G(t)$ for all $t \in \mathbb{R}$. Using these properties, it follows from the expression of the mixed density $f$

$$
f(x)=\pi^{0} g\left(x-u_{1}^{0}\right)+\left(1-\pi^{0}\right) g\left(x-u_{2}^{0}\right)
$$

the following formulae:

$$
\begin{gathered}
\frac{f\left(u_{1}^{0}-t\right)+f\left(u_{1}^{0}+t\right)}{2}=\pi^{0} g(t)+\left(1-\pi^{0}\right) \bar{g}_{s}(t), \\
\frac{f\left(u_{2}^{0}-t\right)+f\left(u_{2}^{0}+t\right)}{2}=\left(1-\pi^{0}\right) g(t)+\pi^{0} \bar{g}_{s}(t), \\
F\left(\mu_{2}^{0}+t\right)-F\left(\mu_{1}^{0}+t\right)=\pi^{0} G(s+t)+\left(1-\pi^{0}\right) G(t)-\left(\pi^{0} G(t)+\left(1-\pi^{0}\right) G(-s+t)\right) \\
=\left(1-2 \pi^{0}\right) G(t)+\pi^{0} G(s+t)-\left(1-\pi^{0}\right) G(t-s),
\end{gathered}
$$




$$
F\left(\mu_{2}^{0}-t\right)-F\left(\mu_{1}^{0}-t\right)=\left(1-2 \pi^{0}\right) G(-t)+\pi^{0} G(t-s)-\left(1-\pi^{0}\right) G(-t-s) .
$$

so that

$$
\begin{aligned}
& F\left(\mu_{2}^{0}+t\right)-F\left(\mu_{1}^{0}+t\right)+F\left(\mu_{2}^{0}-t\right)-F\left(\mu_{1}^{0}-t\right) \\
& =1-2 \pi^{0}+\pi^{0}-G(-t-s)+\pi^{0}-G(t-s) \\
& =1-G(-t-s)-G(t-s) \\
& =G(t+s)-G(t-s) .
\end{aligned}
$$

for all $t \in \mathbb{R}$.

Moment calculations In the sequel, let $\sigma^{2}$ and $m_{4}$ denote the variance and the forth (non-centered) moment of $g$ respectively. We have that

$$
\begin{aligned}
\int_{\mathbb{R}} t^{2}(g(t-s)+g(t+s)) d t & =\int_{\mathbb{R}}(t-s+s)^{2} g(t-\delta) d t+\int_{\mathbb{R}}(t+s-s)^{2} g(t+s) d t \\
& =\int_{\mathbb{R}}\left((x+s)^{2}+(x-s)^{2}\right) g(x) d x \\
& =2 \int_{\mathbb{R}}\left(x^{2}+s^{2}\right) g(x) d x=2\left(\sigma^{2}+s^{2}\right), \\
\int_{\mathbb{R}} t^{4}(g(t-s)+g(t+s)) d t & =\int_{\mathbb{R}}\left((x+s)^{4}+(x-s)^{4}\right) g(x) d x \\
& =\int_{\mathbb{R}}\left(2 x^{4}+12 x^{2} s^{2}+2 s^{4}\right) g(x) d x \\
& =2\left(m_{4}+6 \sigma^{2} s^{2}+s^{4}\right),
\end{aligned}
$$

and using integration by parts

$$
\begin{aligned}
\int_{\mathbb{R}}(G(t+s)-G(t-s)) d t & =\int_{\mathbb{R}} t(g(t-s)-g(t+s)) d t \\
& =\int_{\mathbb{R}}(x+s) g(x) d x-\int_{\mathbb{R}}(x-s) g(x) d x=2 s \\
\int_{\mathbb{R}} t^{2}(G(t+s)-G(t-s)) d t & =\frac{1}{3} \int_{\mathbb{R}} t^{3}(g(t-s)-g(t+s)) d t \\
& =\frac{1}{3} \int_{\mathbb{R}}\left((x+s)^{3}-(x-s)^{3}\right) g(x) d x \\
& =\frac{1}{3} \int_{\mathbb{R}}\left(6 x^{2} s+2 s^{3}\right) g(x) d x \\
& =2 \sigma^{2} s+\frac{2}{3} s^{3}
\end{aligned}
$$


and

$$
\begin{aligned}
\int_{\mathbb{R}} t^{4}(G(t+s)-G(t-s)) d t & =\frac{1}{5} \int_{\mathbb{R}} t^{5}(g(t-s)-g(t+s)) d t \\
& =\frac{1}{5} \int_{\mathbb{R}}\left((x+s)^{5}-(x-s)^{5}\right) g(x) d x \\
& =\frac{1}{5} \int_{\mathbb{R}}\left(10 x^{4} s+20 x^{2} s^{3}+2 s^{5}\right) g(x) d x \\
& =2\left(m_{4} s+2 \sigma^{2} s^{3}+\frac{s^{5}}{5}\right) .
\end{aligned}
$$

Computing the entries of $\mathbb{A}$ We give here the entries of $\mathbb{A}$ (when they have a closed expression) for the examples treated in Section 4.

1. $g^{0}(t)=(\sqrt{2 \pi})^{-1} e^{-t^{2} / 2}$. We can show that

$$
\begin{aligned}
& \mathbb{A}_{11}=4\left(\pi^{0}\right)^{2}\left\{\frac{1}{2 \sqrt{\pi}}\left(\left(\pi^{0}\right)^{2}+\frac{\left(1-\pi^{0}\right)^{2}}{2}\right)+\frac{\pi^{0}\left(1-\pi^{0}\right)}{\sqrt{\pi}} e^{-s^{2} / 4}+\frac{\left(1-\pi^{0}\right)^{2}}{4 \sqrt{\pi}} 2^{-s^{2}}\right\}, \\
& \mathbb{A}_{22}=4\left(1-\pi^{0}\right)^{2}\left\{\frac{1}{2 \sqrt{\pi}}\left(\left(1-\pi^{0}\right)^{2}+\frac{\left(\pi^{0}\right)^{2}}{2}\right)+\frac{\pi^{0}\left(1-\pi^{0}\right)}{\sqrt{\pi}} e^{-s^{2} / 2}+\frac{\left(\pi^{0}\right)^{2}}{4 \sqrt{\pi}} 2^{-s^{2}}\right\}, \\
& \mathbb{A}_{12}=4 \pi^{0}\left(1-\pi^{0}\right)\left\{\frac{3 \pi^{0}\left(1-\pi^{0}\right)}{4 \sqrt{\pi}}+\frac{1}{2 \sqrt{\pi}}\left(\left(\pi^{0}\right)^{2}+\frac{\left(1-\pi^{0}\right)^{2}}{2}\right) e^{-s^{2} / 4}+\frac{\pi^{0}\left(1-\pi^{0}\right)}{4 \sqrt{\pi}} e^{-s^{2}}\right\} .
\end{aligned}
$$

where $s=\mu_{2}^{0}-\mu_{1}^{0}$.

2. $g^{0}(t)=2^{-1} \mathbb{1}_{t \in[-1,1]}$. We can show that

$$
\begin{gathered}
\mathbb{A}_{11}=4\left(\pi^{0}\right)^{2}\left\{\frac{1}{2}\left(\left(\pi^{0}\right)^{2}+\frac{\left(1-\pi^{0}\right)^{2}}{2}\right)+\frac{\pi^{0}\left(1-\pi^{0}\right)}{2}(2-s) \mathbb{1}_{s \leq 2}+\frac{\left(1-\pi^{0}\right)^{2}}{4}(1-s) \mathbb{1}_{s \leq 1}\right\}, \\
\mathbb{A}_{22}=4\left(1-\pi^{0}\right)^{2}\left\{\frac{1}{2}\left(\left(1-\pi^{0}\right)^{2}+\frac{\left(\pi^{0}\right)^{2}}{2}\right)+\frac{\pi^{0}\left(1-\pi^{0}\right)}{2}(2-s) \mathbb{1}_{s \leq 2}+\frac{\left(\pi^{0}\right)^{2}}{4}(1-s) \mathbb{1}_{s \leq 1}\right\}, \\
A_{33}=\left(\frac{4}{3} s^{3}+2(1-s) s^{2}\right) \mathbb{1}_{s \leq 1}+\mathbb{1}_{s>1}\left(\frac{4}{3}+2(s-1)\right),
\end{gathered}
$$




$$
\begin{aligned}
\mathbb{A}_{13}=-2 \pi^{0}\left\{\pi^{0}\right. & {\left[\left(1+s-\frac{s^{2}}{4}\right) \mathbb{1}_{s \leq 2}+2 \mathbb{1}_{s>2}-1\right] } \\
& \left.+\frac{1-\pi^{0}}{2}\left[\left(1+2 s-s^{2}\right) \mathbb{1}_{s \leq 1}+2 \mathbb{1}_{s>1}\right]\right\}, \\
\mathbb{A}_{13}=-2\left(1-\pi^{0}\right)\{ & \left(1-\pi^{0}\right)\left[\left(1+s-\frac{s^{2}}{4}\right) \mathbb{1}_{s \leq 2}+2 \mathbb{1}_{s>2}-1\right] \\
+ & \left.\frac{\pi^{0}}{2}\left[\left(1+2 s-s^{2}\right) \mathbb{1}_{s \leq 1}+2 \mathbb{1}_{s>1}\right]\right\} .
\end{aligned}
$$

\section{References}

Hunter, D. R., Wang, S. and Hettmansperger, T. P. (2007). Inference for mixtures of symmetric distributions. Ann. Statist. 35 224-251.

URL http://dx.doi.org/10.1214/009053606000001118

Shorack, G. R. and Wellner, J. A. (1986). Empirical processes with applications to statistics. Wiley Series in Probability and Mathematical Statistics: Probability and Mathematical Statistics, John Wiley \& Sons, Inc., New York.

VAN DER VAart, A. W. and Wellner, J. A. (1996). Weak convergence and empirical processes. Springer Series in Statistics, Springer-Verlag, New York. With applications to statistics. 